\title{
RECONSIDERAÇÕES SOBRE O TEMPO DE CONSOLIDAÇÃO DAS FRATURAS NA PICNODISOSTOSE
}

\section{RECONSIDERATIONS REGARDING TIME OF FRACTURE HEALING IN PYCNODYSOSTOSIS}

Flávio Dorcilo Rabelo', Carlos Henrique Ribeiro do Prado², Flávio Leão Rabelo ${ }^{3}$, Letícia Martins ${ }^{4}$

\section{RESUMO}

Objetivo: Discutir o que vem sendo descrito na literatura até então a respeito do tempo de consolidação das fraturas na picnodisostose. Métodos: Treze novos casos foram estudados por questão de disponibilidade de prontuários e exames radiográficos, totalizando 44 fraturas englobando pacientes avaliados no período de novembro de 1970 a agosto de 2004, no Hospital Ortopédico de Goiânia. Pesquisa em campo, acompanhamento clínico simultâneo, por novas fraturas, de duas pacientes e avaliação retrospectiva de prontuários foram feitos, com base em se determinar o número de fraturas totais de cada paciente e quais destas tinham viabilidade para esta pesquisa. O grupo de pacientes compõe-se de três mulheres e dois homens com idade média de 51,4 anos. A tíbia foi o osso mais acometido, seguido pelo fêmur. Foram excluídas as fraturas cujo acompanhamento tenha sido feito em outro serviço. Resultados: Das 12 fraturas consideradas plenas para o estudo, nove aconteceram em fêmures (seis no fêmur $\mathrm{E}$ e três no fêmur D; uma em tíbia (D); uma em clavícula (D) e uma em ulna (E)). Dentre as 12 fraturas, oito evoluíram com pseudartrose em um tempo médio de 29,25 meses; três consolidaram bem em uma média de 5,83 meses e um paciente evoluiu com retardo de consolidação em apenas dois meses. Conclusão: Associadas à pesquisa gênica e estudos micromorfológicos, aguarda-se novos estudos para reconfirmação diagnóstica numa entidade clínica tão rara.

Descritores - Pseudartrose; Doenças do Desenvolvimento Ósseo; Fraturas Espontâneas; Consolidação da Fratura

\section{ABSTRACT}

Objective: To discuss what has been described so far in the literature regarding the time of consolidation of fractures in Pycnodysostosis. Materials and Methods: Thirteen new cases were studied, as a matter of the availability of medical records and radiographic examinations, totaling 44 patients in the period from November 1970 to August 2004 in the Hospital Ortopédico de Goiânia. Field research and simultaneous clinical monitoring for new fractures in two patients, and the retrospective evaluation of medical records were made on the basis of determining the total number of fractures for each patient to determine which of these were viable for this study. The group of patients is composed of three women and two men aged 51.4 years. The tibia was the bone most affected, followed by the femur. Fractures the follow-up for which had been done in another facility were excluded. Results: Of the 12 fractures fully considered for the study, nine occurred in femurs (six in the femur $E$ and three in the femur D; one in the tibia (D); one in the clavicle (D); and one in the ulna (E)). Among the 12 fractures, eight developed pseudoarthrosis at an average of 29.25 months, three consolidated well in an average of 5.83 months, and one patient progressed with delayed consolidation in just 2 months. Conclusion: In combination with genetic and micromorphological studies, we await further studies to reconfirm the diagnosis of such a rare clinical entity.

Keywords - Pseudarthrosis; Bone Diseases, Developmental; Fractures, Spontaneous; Fracture Healing

1 - Chefe do Serviço de Residência Médica do Hospital Ortopédico de Goiânia.

2 - Consultor do Grupo de Oncologia Ortopédica do Hospital Ortopédico de Goiânia.

3 - Chefe do Grupo de Oncologia Ortopédica do Hospital Ortopédico de Goiânia.

4 - Fisioterapeuta Especialista do Hospital Ortopédico de Goiânia e Mestre em Ciências da Saúde pela Universidade de Brasília.

Trabalho realizado no Hospital Ortopédico de Goiânia Ltda

Correspondência: Av. L no 470, Setor Aeroporto - Goiânia/GO CEP. 74075-030. E-mail: coremehog@gmail.com

Trabalho recebido para publicação: 04/09/09, aceito para publicação: 28/05/10. 


\section{INTRODUÇÃO}

A picnodisostose é uma doença do grupo das displasias ósseas crânio-tubulares, de distribuição universal e elevada associação com a consanguinidade, graças à sua herança genética. Durante a vida, os ossos dos pacientes afetados tornam-se cada vez mais escleróticos, basicamente devido a uma falha na atividade osteoclástica por mutações no gene da enzima catepsina $\mathrm{K}$, localizado no cromossomo $1 \mathrm{q} 21^{(1-4)}$.

Portanto, a densidade óssea aumentada, nesses pacientes, leva a fraturas por trauma mínimo. Estas geralmente ocorrem no membro inferior e o tratamento atual é principalmente direcionado aos sintomas, com ênfase na prevenção de fraturas ${ }^{(5,6)}$. E deformidades dos ossos longos por consolidação viciosa também são comuns.

Esta displasia esquelética caracteriza-se por baixa estatura importante, membros curtos, osteoesclerose, com tendência a fraturas ósseas múltiplas. As clavículas são displásicas, com aplasia parcial distal do acrômio ${ }^{(3,5,7)}$.

A principal característica radiográfica é um aumento da densidade óssea de todo o esqueleto; esclerose difusa; canal medular, embora pequeno e imperfeito, está presente e sempre com evidência de hematopoiese $\mathrm{e}^{(5)}$.

Ainda de acordo com as características radiográficas, as clavículas são displásicas, hipoplásicas ou aplásicas na sua porção acromial. E no quadril, as cabeças femorais podem ser achatadas; os colos femorais curtos e deformados, com tendência à coxa valga ${ }^{(7)}$.

Portanto, o objetivo deste trabalho é, de certa forma, discutir o que vem sendo descrito na literatura sobre o tempo de consolidação das fraturas na picnodisostose. Certas referências são confusas na exposição do tema, outras são categoricamente mantenedoras da posição de demanda normal de cura das fraturas nesta patologia e algumas referendam a posição deste trabalho, mas sem a devida relevância que o tema requer.

A experiência clínica associada ao estudo molecular recente da picnodisostose evidencia que mais um problema está a se adicionar ao extenso rol clínico desta doença: o retardo de consolidação e a pseudartrose como entidades habituais.

\section{MATERIAIS E MÉTODOS}

Trata-se de um estudo descritivo retrospectivo, no qual foram selecionados 13 novos casos de picnodisostose. Por questão de disponibilidade de prontuários e exames radiográficos, assim como acesso aos pacientes, cinco casos, totalizando 44 fraturas e englobando pacientes avaliados no período de novembro de 1970 a agosto de 2004, no Hospital Ortopédico de Goiânia, fazem parte deste trabalho.

Pesquisa em campo, acompanhamento clínico simultâneo, por novas fraturas, de duas pacientes e avaliação retrospectiva de prontuários foram feitos, com base em se determinar o número de fraturas totais de cada paciente e quais destas fraturas tinham validade para esta pesquisa, ou seja, quais demandavam tempo e qualidade seriada de acompanhamento, com prontuários abrangentes e exames radiográficos sequenciais ou finais de boa qualidade para determinação da consolidação, retardo de consolidação ou pseudartrose.

Nenhum outro exame de imagem fazia parte da avaliação dos pacientes. Dois exames anatomopatológicos antigos referentes à paciente, dois apenas confirmavam a característica esclerótica e presença de hematopoiese, além de excluir malignidade, sem mais nada acrescentar.

Os critérios de inclusão abrangem: idade do paciente, sexo, cor, total de fraturas, fraturas válidas para o estudo, tipo de tratamento e duração do acompanhamento, clínico ou cirúrgico. Os casos que não abrangiam tais quesitos ou que, de uma maneira, não puderam ser incluídos em uma questão tão restrita de uma patologia tão rara, são apenas citados na confecção do parentesco entre os membros integrantes deste estudo e fazem parte de outro trabalho em preparação, que visa o relato destes casos e seu mapeamento genético.

A etiologia das fraturas, inicialmente postada como critério de inclusão, posteriormente se mostrou desnecessária, devido à totalidade das fraturas comporem traumas leves, como quedas de própria altura ou fraturas por estresse. Obviamente, todas são classificadas como fraturas patológicas.

Foram excluídas aquelas fraturas cujo acompanhamento tenha sido feito em outro serviço, fraturas diagnosticadas como achado clínico e que não incitaram o paciente a procurar auxílio médico no momento do trauma e aquelas fraturas cuja raridade de exames radiográficos não nos permitia mostrar a evolução do caso. Assim, 12 fraturas foram acompanhadas no período de novembro de 1970 a agosto de 2004 no serviço presente, perfazendo uma média de 59,08 meses de acompanhamento, variando de dois a 187 meses. Radiografias com intervalos, em média, bimestrais e prontuário completo com tratamento proposto e evolução prognóstica de cura ou não união estavam presentes em todos esses casos.

O grupo de pacientes compõe-se de três mulheres e dois homens. A idade média destes pacientes é de 
51,4 anos, variando de 39 a 64 anos. Três pacientes fazem parte da mesma família, dois são irmãos. Duas pacientes não têm parentesco com a família descrita, nem entre si. Quatro pacientes são brancos. Uma paciente é negra (Tabela 1).

Tabela 1 - Caracterização dos pacientes de acordo com a idade, sexo e cor.

\begin{tabular}{c|c|c|c|c}
\hline Sumário dos pacientes & & & & \\
\hline № & Paciente & Idade (anos) & Sexo & Cor \\
\hline 1 & MLV & 51 & F & Branca \\
\hline 2 & IMSS & 39 & F & Branca \\
\hline 3 & VSN & 47 & M & Branca \\
\hline 4 & MPS & 64 & F & Negra \\
\hline 5 & HNS & 56 & M & Branca \\
\hline
\end{tabular}

Fonte: Hospital Ortopédico de Goiânia.

Somados, esses pacientes apresentaram, ao longo da vida, 44 fraturas relatadas, confirmadas em prontuário e em exame radiográfico.

A tíbia foi o osso mais acometido, com 25 fraturas. Seguida pelo fêmur: 14 fraturas; clavícula e acrômio, duas fraturas cada; e ulna, com uma fratura (Figura 1). Não houve predominância de lado acometido, com 21 fraturas ocorrendo à esquerda (E) e 23 fraturas à direita (D) (Figura 2).

Paciente e osso acometido, tempo de seguimento específico de cada uma dessas 12 fraturas, assim como parentesco com outros portadores de picnodisostose excluídos do trabalho são apresentados na Tabela 2.

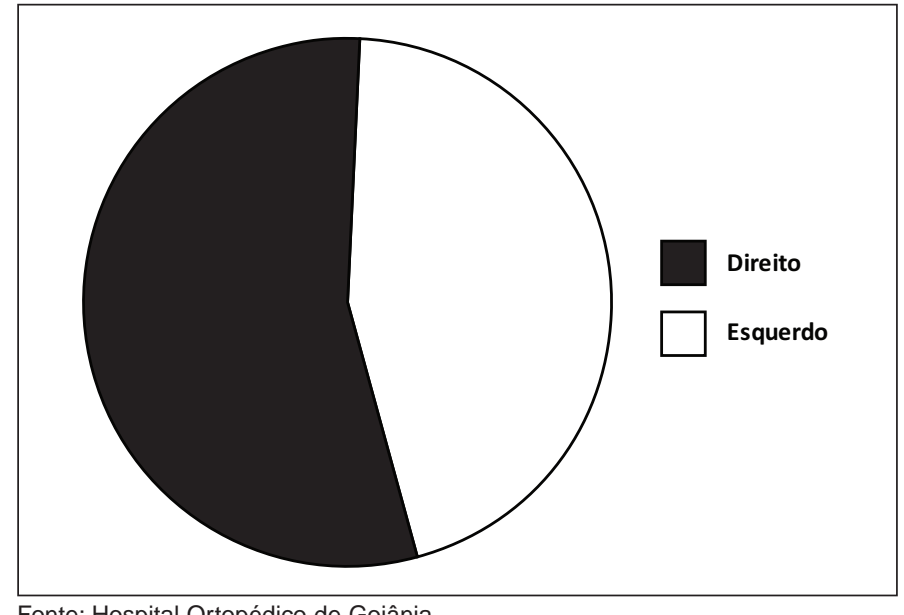

Fonte: Hospital Ortopédico de Goiânia.

Figura 1 - Caracterização da amostra do estudo de acordo com o osso acometido, Goiânia, GO, 2010.

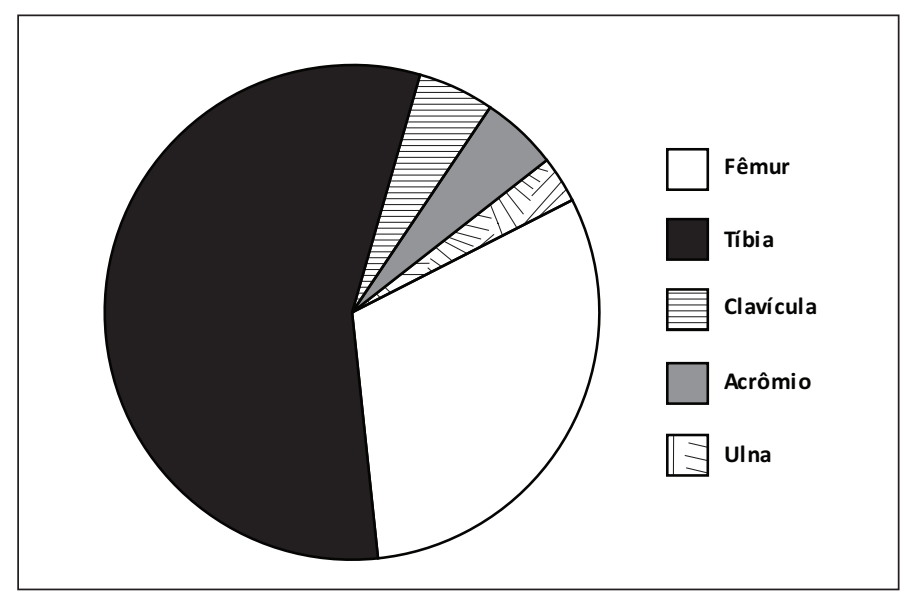

Fonte: Hospital Ortopédico de Goiânia.

Figura 2 - Caracterização da amostra do estudo de acordo com o lado acometido, Goiânia, GO, 2010.

Tabela 2 - Correlação dos pacientes com picnodisostose de acordo com a fratura válida e parentes acometidos.

\begin{tabular}{|c|c|c|c|c|}
\hline Paciente & Osso fraturado & Tempo de seguimento & Parentes acometidos & Observações \\
\hline \multirow{4}{*}{1} & Fêmur D & $3 \mathrm{M}$ & \multirow{4}{*}{2 irmãos } & \\
\hline & Fêmur E & $108 \mathrm{M}$ & & \\
\hline & Ulna E & $4 \mathrm{M}$ & & \\
\hline & Clavícula D & $120 \mathrm{M}$ & & \\
\hline \multirow{2}{*}{2} & Fêmur E & $187 \mathrm{M}$ & Irmã № 3 & 3 irmãs e pais normais \\
\hline & Fêmur D & $28 \mathrm{M}$ & Prima em $2^{\circ}$ grau № $5+6$ primos em $2^{\circ}$ grau & \\
\hline \multirow{2}{*}{3} & \multirow{2}{*}{ Tíbia D } & \multirow{2}{*}{$2 \mathrm{M}$} & Irmão № 2 & 3 irmãs e pais normais \\
\hline & & & Primo em $2^{\circ}$ grau № $5+6$ primos em $2^{\circ}$ grau & \\
\hline \multirow{4}{*}{4} & Fêmur D & $108 \mathrm{M}$ & \multirow{4}{*}{ Paciente adotada. Não conhece parentes } & \\
\hline & Fêmur $\mathrm{E}$ & $18 \mathrm{M}$ & & \\
\hline & Fêmur E * & $56 \mathrm{M}$ & & \multirow{2}{*}{$\begin{array}{c}{ }^{*} \text { Fratura do material de } \\
\text { síntese associada }\end{array}$} \\
\hline & Fêmur $E^{*}$ & $49 \mathrm{M}$ & & \\
\hline \multirow{3}{*}{5} & \multirow{3}{*}{ Fêmur E } & \multirow{3}{*}{$26 \mathrm{M}$} & 1 irmão e pai & \multirow{3}{*}{3 irmãos e pais normais } \\
\hline & & & Tia paterna & \\
\hline & & & Primo em $2^{\circ}$ grau № 2 e $3+5$ primos em $2^{\circ}$ grau & \\
\hline
\end{tabular}




\section{RESULTADOS}

Das 12 fraturas consideradas plenas para o estudo, nove aconteceram em fêmures (seis no fêmur $\mathrm{E}$ e três no fêmur D); uma em tíbia (D); uma em clavícula (D) e uma em ulna (E) (Figura 3). Totalizam sete fraturas no lado esquerdo e cinco no lado direito.

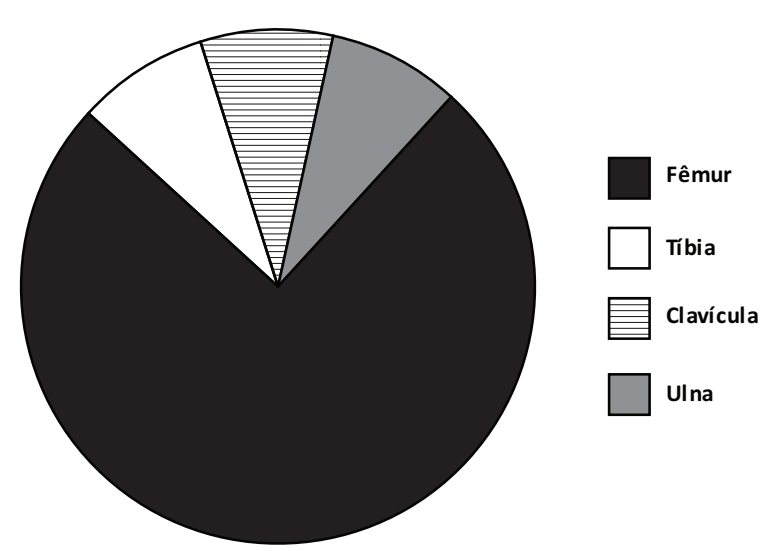

Fonte: Hospital Ortopédico de Goiânia.

Figura 3 - Caracterização da amostra do estudo de acordo com as fraturas válidas, Goiânia, GO, 2010.

A paciente 1 foi inicialmente vista neste hospital em novembro de 1970, por uma fratura diafisária do fêmur D (Figura 4).

Os demais pacientes, respectivas fraturas e variáveis encontram-se expostas na Tabela 3.

As fraturas descritas seguiram tratamento inicial, conforme o preconizado na época, para fraturas habituais. Três casos demandaram a colocação de enxerto
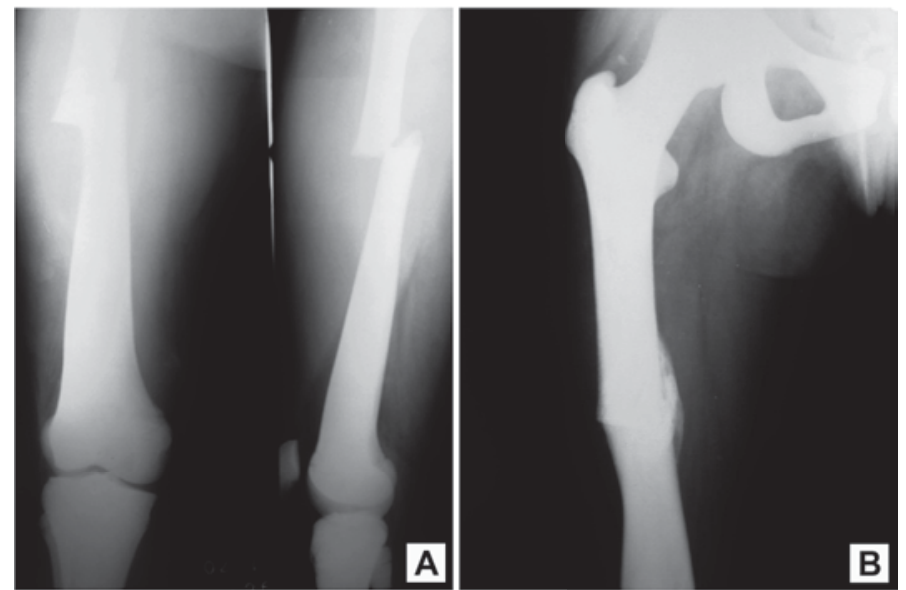

Fonte: Hospital Ortopédico de Goiânia.

Figura 4 - Fratura diafisária do fêmur em paciente do sexo feminino, ocorrida em 1970, aos 17 anos (A) e tratada com tração esquelética e gesso pelvipodálico, mostrando bom calo ósseo dois meses e 14 dias após a fratura (B).

ósseo: dois por opção dos cirurgiões, em fraturas primárias; sendo que em uma (ulna), já havia sido tentado o tratamento conservador, sem sucesso. E um, por refratura associada à fratura do material de síntese. Quatro pacientes foram tratados conservadoramente; sete, cirurgicamente e um paciente aguarda tratamento, neste momento. Das doze fraturas, oito $(66,66 \%)$ evoluíram com pseudartrose em um tempo médio de 29,25 meses (M), variando de três a 62 meses. Três (25\%) consolidaram bem em uma média de 5,83 $\mathrm{M}$, variando de 2,5 a $10 \mathrm{M}$. Um paciente $(8,33 \%)$ evoluiu com retardo de consolidação em apenas dois meses, com nenhuma formação inicial de calo em tratamento conservador para fratura de $1 / 3$ médio da tíbia.

Tabela 3 - Caracterização dos pacientes com picnodisostose de acordo com as fraturas válida e resultados obtidos, Goiânia.

\begin{tabular}{c|c|c|c|c}
\hline Paciente & Fratura & TS* & Acompanhamento & Tratamento \\
\hline \multirow{4}{*}{1} & Fêmur diáfise D & $3 \mathrm{M}$ & Sob internação & Tração esquelét. + gesso pelvipodálico \\
\cline { 2 - 5 } & Fêmur subTC** E & $108 \mathrm{M}$ & Ambulatorial mensal & Cirúrgico: placa DCP 8 furos + enxerto ósseo \\
\cline { 2 - 5 } & Ulna E $1 / 3$ proximal & $4 \mathrm{M}$ & Ambulatorial semanal & Gesso axilopalmar \\
\cline { 2 - 5 } & Clavícula D $1 / 3$ médio & $120 \mathrm{M}$ & Ambulatorial semanal & Oito gessado \\
\hline \multirow{3}{*}{2} & Fêmur diáfise E & $187 \mathrm{M}$ & Ambulatorial mensal e semestral & Cirúrgico: placa DCP 10 furos \\
\cline { 2 - 5 } & Fêmur diáfise D & $28 \mathrm{M}$ & Ambulatorial mensal & Cirúrgico: placa DCP 10 furos \\
\hline \multirow{3}{*}{3} & Tíbia D $1 / 3$ médio & $2 \mathrm{M}$ & Ambulatorial semanal & Gesso tipo bota \\
\cline { 2 - 5 } & Fêmur subTC D & $108 \mathrm{M}$ & Ambulatorial anual & Cirúrgico: Jewett \\
\cline { 2 - 5 } & Fêmur subTC E & $18 \mathrm{M}$ & Ambulatorial mensal & Cirúrgico: Richards + enxerto ósseo \\
\cline { 2 - 5 } & Fêmur diáfise E & $56 \mathrm{M}$ & Ambulatorial mensal & Aguarda cirurgia \\
\hline \multirow{2}{*}{5} & Fêmur subTC E & $49 \mathrm{M}$ & Ambulatorial mensal & Cirúrgico: Richards \\
\hline
\end{tabular}

*TS: tempo de seguimento/ **subTC: subtrocanteriana.

Fonte: Hospital Ortopédico de Goiânia. 
De oito fraturas que evoluíram com pseudartrose, seis haviam sido tratadas por cirurgia e duas por tratamento conservador. Da mesma forma, nas três fraturas que evoluíram com boa consolidação em tempo hábil, duas foram tratadas por cirurgia e uma conservadoramente.

Duas fraturas que consolidaram bem são de uma mesma paciente (2) (as únicas apresentadas por ela neste estudo). Entretanto seu irmão (paciente 3), apresentou um precoce retardo de consolidação em outro tipo de fratura.

Os demais pacientes evoluíram com um elevado índice de pseudartrose.

\section{DISCUSSÃO}

O tempo de consolidação de uma fratura não pode ser arbitrariamente estabelecido. Não existem dados com respeito à natureza e duração de consolidação de fraturas em picnodisostose ${ }^{(8)}$.

Em ossos normais, uma união é considerada atrasada quando a consolidação não ocorreu na média hábil para o local e tipo de fratura (normalmente de três a seis meses). O diagnóstico de não-união é injustificado até evidência clínica ou radiológica mostrar que a consolidação parou e que a união é altamente improvável. O status final de uma fratura não-unida é a formação de uma pseudartrose. Judet e Judet diferenciaram dois tipos básicos de pseudartroses: as hipertróficas (capazes de uma reação biológica) e as atróficas

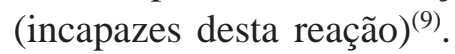

Apesar das causas do retardo de consolidação e da pseudartrose serem desconhecidas, vários fatores sistêmicos e locais contribuem para o seu desenvolvimento, a saber: tipo e local de fratura, se há infecção, tipo de fixação, tempo de imobilização, uso de álcool e tabaco, estado metabólico e nutricional do paciente, osso irradiado ou com alguma alteração constitucional.

Na picnodisostose, a reabsorção óssea é defeituosa, mas não completamente inibida. Na verdade, parece que existe uma alternativa bioquímica, não osteoclástica, para a degradação da matriz óssea: células com atividade de metaloproteinases degradam a matriz deixadas pelos osteoclastos.

Entretanto, o mecanismo, função e forma de degradação das duas vias é distinta e o funcionamento de apenas uma pode alterar o microambiente ósseo, contribuindo inclusive para alterações na função osteoblástica ${ }^{(10)}$, o que pode modificar o fenótipo esclerótico e o padrão de consolidação das frequentes fraturas apresentadas pelos portadores da doença.
A degradação da matriz óssea é um passo fundamental para promover a neoaposição óssea. Recentes estudos têm mostrado inclusive a disfunção osteoclástica associada com um déficit na formação óssea, dependente da idade e de cargas mecânicas ${ }^{(10)}$, ou seja, a disfunção osteoclástica aumenta de tal maneira o volume ósseo, que este paralisa não só a função osteoclástica como, também, a osteoblástica.

A homogeneidade da matriz óssea é severamente afetada e a estrutura do osso lamelar sofre inúmeras consequências, com as trabéculas não mais formando linhas na direção do estresse mecânico, o que explica a tão contraditória fragilidade óssea da picnodisostose, e com reflexão nas fibrilas de colágeno e seu alinhamento de partículas minerais, que se encontra reduzido influenciando a regeneração óssea e a consolidação das fraturas.

Os resultados de 66\% de pseudartrose nas fraturas patológicas associadas à picnodisostose, encontradas neste estudo, embasam esta teoria e levantam considerações sobre quais os melhores métodos de tratamento para estas fraturas e sobre qual o tempo de consolidação esperado em casos tão restritos.

Sabe-se que a utilização de osteossíntese com placas e parafusos só é eficiente quando um gap menor ou igual a $1 \mathrm{~mm}$ estiver presente entre os fragmentos e que a consolidação se dá sem a formação de calo, por contato cortical primário(11), o que desfavorece esta forma de tratamento na picnodisostose.

Entretanto, a utilização de hastes, que levaria à formação do calo ósseo, é difícil em uma patologia com grande alteração da medular (Figura 5) e do compri-

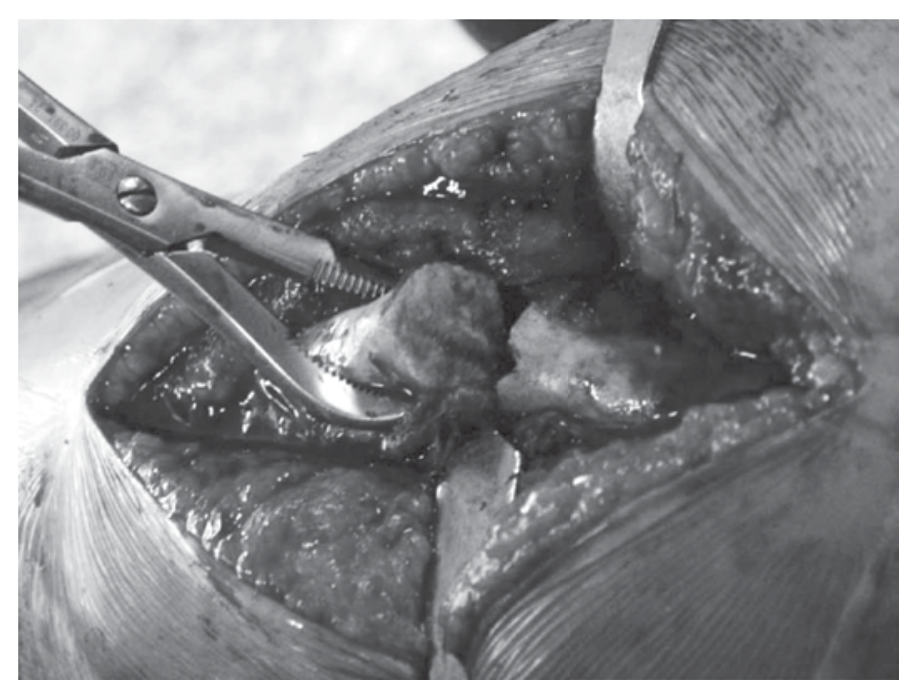

Fonte: Hospital Ortopédico de Goiânia

Figura 5 - Aspecto macroscópico de ausência medular na picnodisostose que, ao contrário da osteopetrose, não se confirma na radiografia e na prática. 
mento dos $\operatorname{ossos}^{(12)}$. O tratamento conservador é, sabidamente, produtor de pseudartroses ${ }^{(9)}$ e incapacitante ao extremo, no tratamento de fraturas dos ossos longos do membro inferior em adultos.

O uso de enxerto ósseo nesta patologia ainda não está bem estabelecido. Não se sabe até que ponto haveria degradação de matriz óssea alógena e testes com proteínas morfogenéticas do osso ainda carecem de bons resultados em avaliações no osso normal.

\section{CONCLUSÕES}

Apesar de inúmeros trabalhos afirmarem que o tempo de consolidação na picnodisostose é normal, dúvidas foram levantadas a respeito do tema e com o passar do tempo. Pareceu óbvio, com a experiência clínica enfrentada com tais pacientes que, apesar dos tratamentos empregados e falhas ocasionadas, o padrão de consolidação na picnodisostose, seja ela endosteal ou periosteal, ditava seu próprio ritmo. Um ritmo lento, com tendência a pseudartrose e altamente dependente das funções blásticas e clásticas intrinsicamente alteradas. Depende agora da sequência de estudos micromorfológicos e com materiais de síntese, mapeamento genético e mais estudos retrospectivos, já que os prospectivos enfrentariam a barreira da raridade na amostragem, para reconfirmação da certeza.

\section{REFERÊNCIAS}

1. Petersen JN, Haagerup A, Nørholt SE, Børglum AD, Mosekilde L. Pyknodysostose: diagnostiske overvejelser og behandling af to patienttilfælde. Tandlægebladet. 2002;106(5):362-8.

2. Emami-Ahari Z, Zarabi M, Javid B.. Pycnodysostosis. J Bone Joint Surg Br. 1969;51(2):307-12.

3. Motyckova G, Fisher DE. Pycnodysostosis: role and regulation of cathepsin K in osteoclast function and human disease. Curr Mol Med. 2002;2(5):407-21.

4. Gelb BD, Shi GP, Chapman HA, Desnick RJ. Pycnodysostosis, a lysosomal disease caused by cathepsin K deficiency. Science. 1996;273(5279):1236-8.

5. Elmore SM. Pycnodysostosis: a review. J Bone Joint Surg Am. 1967;49:153-62.

6. Oliveira CL, Oliveira LN, Cruz RL, Braune AS, Tonomuras E. Diferenças clínicas e radiológicas entre portadores de osteopetrose e picnodisostose. Rev Bras Cir Craniomaxilofac. 2010;13(1):49-54.

7. Quezado Junior Q, Montenegro RM, Araripe FF, Correa RV, Montenegro RM. Picno- disostose: relato de dois casos. Arq Bras Endocrinol Metab. 2003;47(1):95-101.

8. Jiya TU, Hindrik M, Kleipool, Ham J. Diaphyseal femur fracture in pycnodysostosis treated with pennig wrist external fixator: a case study. J Trauma. 2006;49(5):477-9.

9. LaVelle DG. "Delayed union and nonunion of fractures". In: Canale ST. Campbell's Operative Orthopaedics. Philadelphia: Mosby; 2003. p. 3125-65.

10. Fratzl-Zelman N, Valenta A, Roschger P, Nader A, Gelb BD, Fratzl P, et al. Decreased bone turnover and deterioration of bone structure in two cases of pycnodysostosis. J Clin Endocrinol Metab. 2004;89(4):1538-47.

11. Cornell CN, Lane JM. Newest factors in fracture healing. Clin Orthop Relat Res. 1992;(277):297-311.

12. Kundu ZS, Marya KM, Devgan A, Yadav V, Rohilla S. Subtrochanteric fractures managed by intramedullary nail in a patient with pycnodysostosis. Joint Bone Spine. 2004;71(2):154-7. 